Niveles de ansiedad de estudiantes frente a situaciones de exámenes:

\title{
Cuestionario CAEX
}

\section{Anxiety levels of students facing exam situations: CAEX questionnaire}

\author{
Sofia Sairitupac Santana \\ Universidad César Vallejo, Lima, Perú \\ ORCID: https://orcid.org/0000-0002-0779-3854 \\ Roxana Patricia Varas Loli \\ Universidad César Vallejo, Lima, Perú \\ ORCID: https://orcid.org/0000-0003-1426-6558 \\ José Nieto-Gamboa \\ Universidad César Vallejo, Lima, Perú \\ ORCID: https://orcid.org/0000-0002-1844-8765 \\ Bertha Silva Narvaste \\ Universidad César Vallejo, Lima, Perú \\ ORCID: https://orcid.org/ 0000-0002-2926-6027 \\ Máximo Abel Rodriguez Taboada \\ Universidad de San Martin de Porres, Lima, Perú \\ ORCID: https://orcid.org/0000-0002-8035-2369
}

Received 03-24-20 Revised 05-30-20 Accepted 08-08-20 On line 10-27-20

*Correspondence

Email: ssairitupac@ucv.edu.pe
Cite as:

Sairitupac Santana. S., Varas Loli, R., Nieto-Gamboa, J., Silva Narvaste, B., \& Rodriguez Taboada, M. (2020). Niveles de ansiedad de estudiantes frente a situaciones de exámenes: Cuestionario CAEX.

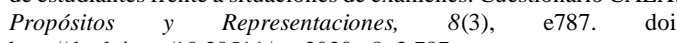
http://dx.doi.org/10.20511/pyr2020.v8n3.787 


\section{Resumen}

El propósito del estudio fue comparar los niveles ansiedad de estudiantes secundarios de quinto año de secundaria de dos instituciones educativas privadas. El estudio es de un enfoque cuantitativo, tipo de estudio sustantiva, de nivel descriptivo comparativo y de diseño no experimental transversal, la población estuvo constituida por 400 estudiantes de dos colegios privados, los estudiantes en referencia cursan el quinto año de secundaria. El instrumento utilizado fue el cuestionario de evaluación de problemas de ansiedad ante los exámenes (CAEX). cuestionario elaborado específicamente para recoger la variedad de respuestas motoras, verbales, cognitivas o fisiológicas que suelen acompañar estos problemas, junto con la tipología de exámenes más frecuentes. Como consecuencia del estudio podemos concluir según el valor del estadístico $U$ de Mann Whitney con p valor $<0,05$, nos indica que existen diferencias significativas en los niveles de ansiedad de los estudiantes de las dos Instituciones educativa privadas en estudio, concluyendo que los estudiantes de la dos Instituciones Educativas privadas presentan niveles altos grados de ansiedad ante los examenes ,situación que puede afectar su estado emocional que son hasta peligrosos para la salud (Grave presencia de ansiedad).

Palabras clave: ansiedad ante los exámenes, preocupaciones, reacciones fisiológicas, situaciones, evitación ante los exámenes.

\section{Summary}

The purpose of the study was to compare the anxiety levels of fifth-year high school students from two private educational institutions. The study is of a quantitative approach, type of substantive study, comparative descriptive level and non-experimental crosssectional design, the population consisted of 400 students from two private schools, the reference students are in the fifth year of secondary school. The instrument used was the Test Anxiety Problem Assessment Questionnaire (CAEX). Questionnaire specifically developed to collect the variety of motor, verbal, cognitive or physiological responses that usually accompany these problems, together with the most frequent type of examinations. As a consequence of the study, we can conclude according to the value of the Mann Whitney $\mathrm{U}$ statistic with $\mathrm{p}$ value $<0.05$, it indicates that there are significant differences in the anxiety levels of the students of the two private educational institutions under study, concluding that the students of the two private educational institutions present high levels of anxiety before the exams, a situation that can affect their emotional state that are even dangerous to health (Serious presence of anxiety).

Key words: test anxiety, concerns, physiological reactions, test situations.

\section{Introducción}

La ansiedad es un estado de ánimo que se caracteriza por presentar preocupaciones de diferente intensidad según la individualidad de la persona y/o de las situaciones que la generan. Conductualmente las personas ansiosas presentan dificultades en la capacidad de indecisión, evitación y búsqueda de confianza, además de inquietud o impaciencia, fatigabilidad, dificultades para concentrarse o dejar la mente en blanco, irritabilidad, tensión muscular, alteraciones del sueño (dificultad para conciliar o mantener el sueño o un sueño reparador.

Se han elaborado varios modelos teóricos para explicar la relación entre la ansiedad ante los exámenes y la ejecución en los mismos. Desde un modelo conductual 
se supone una inhibición de conductas académicas bajo una situación de castigo, con los componentes condicionados o emociones habituales en una situación de ansiedad. Desde una perspectiva cognitiva se incluyen la existencia de pensamientos negativos, dificultad en la resolución de problemas, déficits atencionales y baja auto estima como causa de esta problemática (Gutierrez-Calvo, 1984, 1986).

La ansiedad ante los exámenes es un estado emocional que con el tiempo puede ser un trastorno de personalidad, este estado de ánimo es experimentado por numerosos estudiantes a lo largo de su vida académica, al enfrentarse a situaciones sociales en las que sus aptitudes personales son evaluadas. La ansiedad, está íntimamente relacionada con el malestar psicológico y el bajo rendimiento académico, y se encuentra determinada por una interacción compleja y dinámica entre factores objetivos que rodean la situación de evaluación, variables subjetivas familiares y sociales del estudiante (Francisquelo \& Furlan, 2016).

La ansiedad ante los exámenes es definida como una tendencia emocional que sufre el estudiante frente a situaciones de evaluaciones que las considera preocupantes y/o amenazantes, presentan además miedo al fracaso, presentan además reacciones fisiológicas, como taquicardia, sudoración, enuresis, entre otros aspectos que según el tipo de personalidad (Rodríguez, Canedo, Cerviño, \& Enríquez, 2019).

Los exámenes (evaluaciones) producen ansiedad en los estudiantes, porque predisponen al alumnado a que actúe con bastante ansiedad y que pueden repercutir en su rendimiento académico, y auto estima personal, que inclusive los pueden llevar a la frustración, miedos y abandono de sus objetivos (Casari, Anglada, \& Daher, 2014)

Delgado, Castro \& Pérez (2020) manifiestan que al conocer que el alumno está ansioso, este debe tener en una primera instancia sesiones de tutoría y si el caso requiere solicitar la intervención del psicólogo. Profesional que diseñara programas a fin de ayudar al estudiante en el proceso de su desarrollo de enseñanza aprendizaje.

La importancia del estudio de la ansiedad ante los exámenes en poblaciones adolescentes de estudiantes peruanos se ve justificado debido a que, al no ser tratado a tiempo y el aumento de estímulos frustrantes, puede perjudicar su salud mental. Además, se sabe que durante la pubertad se experimenta la ansiedad como resultado del proceso de adaptación a los cambios biológicos, cognitivos, afectivos y sociales, y de la inestabilidad que conlleva la búsqueda de la propia individualidad, y la conciencia de los límites y recursos propios. En base a lo expuesto, el objetivo del estudio fue establecer las diferencias en la prevalencia de la ansiedad en función a la edad y sexo en escolares peruanos. En relación a la edad, los niveles de ansiedad altos están presentes en los niños más que en los adolescentes y estos disminuyen conforme van obteniendo mayor edad. Resultados contrarios señalan que los niveles de ansiedad y los trastornos de ansiedad en la infancia son predictores de futuros trastornos de ansiedad cuando lleguen a la adolescencia (Orgilés et al.,2012); mientras que otros estudios indican que tanto en la infancia como en la adolescencia no se encuentran diferencias significativas en relación a la edad (Inglés et al., 2010). De acuerdo con ello, la ansiedad ante los exámenes constituye una experiencia emocional unívoca en la que se distinguen dos componentes. emocionalidad y preocupación (Cassady \& Johnson, 2002; Segool, Von der Embse, Mata, \& Gallant, 2014), que englobarían las tres manifestaciones - cognitiva, emocional y fisiológica - que caracterizan a la ansiedad como constructo genérico (Miguel-Tobal, 1996; Sierra, Ortega, \& Zubeidat, 2003). 
La ansiedad y el descontrol de la misma conlleva a una alta activación fisiológica y emocional acompañada de cefaleas, náuseas, tensión muscular o sensación de pánico. Por su parte, la preocupación se define, como un estado cognitivo caracterizado por un alto grado de pensamientos autorreferidos, autocrítica, miedo a las consecuencias, fracaso, temor a decepcionar a los padres, estados emocionales que desarrolla el estudiante antes, durante y/o inmediatamente después del examen (Boehme, Goetz, \& Preckel, 2017; Lohbeck, Nitkowski, \& Petermann, 2016; Putwain \& Aveyard, 2018; Steinmayr, Crede, McElvany, \& Wirthwein, 2016).

La elevada ansiedad ante los exámenes se relaciona con las dificultades cuando ejecutan y este estado de ánimo repercute en el rendimiento académico. El estudiante con ansiedad se da cuenta de las exigencias de cada situación que amenaza a su ego, conduciéndolo a una autofocalización que va a dividir la atención en los procedimientos al ejecutar las labores académicas y las cogniciones sin importancia que se asocian al "sí mismo", lo que llega a disminuir su rendimiento académico. Furlan, Rosas, Heredia, Piemontesi, \& Illbele, (2009). La ansiedad ante los exámenes, es una característica de personalidad específica que repercute en su atención, concentración y realización de exámenes (Dominguez-Lara, 2016).

Las evaluaciones (examenes) son situaciones que estresan y que son percibidas por los alumnos, denotando que a mayor grado de estrés es mayor la percepción negativa en la eficacia y rendición académica (Jiménez \& Ramírez, 2017).

\section{Factores que influyen en la ansiedad ante los examenes}

Los factores que producen la ansiedad en los estudiantes frente a un examen: a) El rasgo de ansiedad del sujeto, esto es, su carácter más o menos influenciable por la ansiedad. Según sea su rasgo de ansiedad, se puede clasificar a los estudiantes a lo largo de una línea continua que va desde los poco ansiosos a los altamente ansiosos. b) Según la importancia de la prueba. No causa la misma ansiedad un control rutinario, o una prueba parcial con posibilidades de recuperación, que un examen final que implica aprobar o suspender la asignatura y con lleva a pasar al curso siguiente o repetir. Cuando el examen es el último de una oposición y el examinando se juega una plaza, la dosis de ansiedad puede ser muy elevada. c) Factores relacionados con el medio ambiente, tales como: aula desconocida, aspecto de los examinadores, tipo de instrucciones, ruido y calor medioambiental, tiempo disponible, etc., también inciden sobre los sujetos aumentando o disminuyendo su nivel de ansiedad. Con lo anteriormente mencionado se puede afirmar entonces que la ansiedad es una forma de reaccionar emocionalmente ante la presencia de una persona, de un objeto o situación que actúa como amenaza, como son los exámenes, que generan ansiedad en los estudiantes(Bausela ,2005)

\section{Dimensiones de la ansiedad ante los examenes}

La dimensión ansiedad ante los exámenes (Valero, 1999) son las siguientes:

Preocupación: Hace referencia al aspecto cognitivo de la ansiedad, según el autor son los pensamientos y preocupaciones de tipo cognitivo sobre el examen. Por tanto, engloba pensamientos pesimistas e inseguridad de sí mismo, de la situación y de la consecuencia en torno a los exámenes. La preocupación es una reacción automática cuya finalidad es resolver los problemas. Cuando la preocupación se debe a sucesos que tienen pocas posibilidades de solución, se convierte en patológica (Sánchez, 2008). 
Reacciones fisiológicas: Es el conjunto de características fisiológicas que aparecen como respuesta a la ansiedad. Son aquellas respuestas orgánicas provocadas por la tensión excesiva que afronta el estudiante. Su aparición surge ante la activación del sistema nervioso autonómico, dando lugar a los siguientes signos: taquicardia, sudoración, aceleramiento del ritmo cardiaco, entre otros.

Situaciones: Abarca las situaciones y tipos de exámenes más comunes que se rinden para evaluar las competencias educativas. Se evidencia síntomas de afrontamiento a los exámenes según la tipología de los mismos y los factores externos o del entorno académico desencadenantes de una respuesta de ansiedad.

Respuestas de evitación: Hace referencia a los comportamientos de evitación o de escape, el cual es una característica propia de los problemas de ansiedad. es una forma de afrontamiento invertido, en virtud del cual el estudiante que percibe la situación ansiógena.

\section{Método}

El estudio fue de enfoque cuantitativo, tipo sustantiva, nivel descriptivo comparativo y diseño no experimental transversal, teniendo como población 400 estudiantes, constituidas por estudiantes de quinto año de secundaria de dos instituciones educativas privadas. La técnica fue la encuesta y el instrumento utilizado fue el cuestionario de CAEX con un total de 50 Ítems (Valero, 1999), repartidos en 4 factores: respuesta fisiológica (15 ítems), respuesta cognitiva (14 ítems), respuesta de evitación ( 7 ítems) y situaciones de examen (14 ítems) (Hernández y Mendoza, 2018).

\section{Análisis de datos.}

Para el análisis de datos se utilizó el estadístico U de Mann Whitney en la comparación de grupos independientes, ya que se buscó establecer las diferencias entre los niveles de ansiedad de los estudiantes de las dos instituciones educativas.

\section{Resultados}

Tabla 1.

Niveles de ansiedad en la dimensión Situaciones por genero ante los exámenes en los estudiantes de secundaria

\begin{tabular}{|c|c|c|c|c|c|c|}
\hline \multirow[b]{2}{*}{ Sexo } & & & & \multicolumn{2}{|c|}{ Grupo } & \multirow[b]{2}{*}{ Total } \\
\hline & & & & I.E privada 1 & I.E privada 2 & \\
\hline \multirow[t]{8}{*}{ Masculino } & Ansiedad ante los & Leve & Recuento & 51 & 3 & 54 \\
\hline & exámenes & & $\%$ del total & $22,1 \%$ & $1,3 \%$ & $23,4 \%$ \\
\hline & & Moderada & Recuento & 73 & 102 & 175 \\
\hline & & & $\%$ del total & $31,6 \%$ & $44,2 \%$ & $75,8 \%$ \\
\hline & & Grave & Recuento & 0 & 2 & \\
\hline & & & $\%$ del total & $0,0 \%$ & $0,9 \%$ & $0,9 \%$ \\
\hline & Total & & Recuento & 124 & 107 & 231 \\
\hline & & & $\%$ del total & $53,7 \%$ & $46,3 \%$ & $100,0 \%$ \\
\hline \multirow[t]{8}{*}{ Femenino } & Ansiedad ante los & Leve & Recuento & 7 & 0 & 7 \\
\hline & exámenes & & $\%$ del total & $4,1 \%$ & $0,0 \%$ & $4,1 \%$ \\
\hline & & Moderada & Recuento & 67 & 41 & 108 \\
\hline & & & $\%$ del total & $39,6 \%$ & $24,3 \%$ & $63,9 \%$ \\
\hline & & Grave & Recuento & 2 & 52 & 54 \\
\hline & & & $\%$ del total & $1,2 \%$ & $30,8 \%$ & $32,0 \%$ \\
\hline & Total & & Recuento & 76 & 93 & 169 \\
\hline & & & $\%$ del total & $45,0 \%$ & $55,0 \%$ & $100,0 \%$ \\
\hline
\end{tabular}


Según los resultados obtenidos del procesamiento del instrumento aplicado, se aprecia que son los alumnos de la Institución Educativa Particular 1 los que presentan mayores niveles de ansiedad por género es así que son las mujeres los que presentan mayores porcentajes en el nivel Grave presencia de ansiedad con $1.2 \%$, moderado de $39.6 \%$ y $4.1 \%$ de nivel leve; los varones presentan $0 \%$ de nivel grave y $22.1 \%$ en nivel leve.

Mientras que para la Institución educativa particular 2, las mujeres los que obtienen mayores niveles de ansiedad grave con 30.8\%, y los varones presentan el $0.9 \%$ de nivel grave.

Se concluye que existen diferencias entre los valores obtenidos en los niveles de ansiedad por género en las Instituciones de estudio; con las mujeres que presentan mayores grados de ansiedad.

Tabla 2.

Contrastación de hipótesis de comparación del nivel de ansiedad ante los exámenes en estudiantes de secundaria de dos Instituciones Privadas

\begin{tabular}{lllll}
\hline Rangos & & & & \\
\hline & Grupo & $\mathrm{N}$ & \multicolumn{2}{c}{ Rango promedioSuma de rangos } \\
\hline Ansiedad ante los & I.E privada 1 & 200 & 130,25 & 26049,00 \\
exámenes de la Institución & I.E privada 2 & 200 & 270,76 & 54151,00 \\
privada 1 y 2 & Total & 400 & & \\
\hline
\end{tabular}

\begin{tabular}{ll}
\hline Estadísticos de prueba $^{\text {a }}$ & $\begin{array}{l}\text { Ansiedad ante } \\
\text { los exámenes de } \\
\text { la Institución } \\
\text { privada 1 y 2 }\end{array}$ \\
\hline U de Mann-Whitney & 5949,000 \\
W de Wilcoxon & 26049,000 \\
Z & $-12,154$ \\
Sig. asintótica(bilateral) &, 000 \\
a. Variable de agrupación: Grupo \\
\hline
\end{tabular}

El valor del estadístico $U$ de Mann Whitney con $\mathrm{p}$ valor $<0,05$, nos indica que hay diferencias importantes en el grado de ansiedad ante los examenes de los estudiantes de las dos Instituciones educativa privadas en estudio, En cuanto a la institución educativa privada 1 presentó un (Promedio $=135.25)$ a diferencia de la ansiedad ante los exámenes de la institución educativa privada 2 , con un (Promedio $=270.76$, afirmando que existe diferencias significativas de los niveles de ansiedad ante los exámenes en estudiantes de secundaria de dos Instituciones Privadas.

Tabla 3.

Contrastación de hipótesis de comparación de los niveles de preocupación ante los exámenes en estudiantes de secundaria de dos Instituciones Privadas

\begin{tabular}{llrrr}
\hline \multicolumn{5}{c}{ Rangos } \\
\hline & Grupo & N & Rango promedio & Suma de rangos \\
\hline Preocupación & I.E privada 1 & 200 & 148,34 & 29667,00 \\
& I.E privada 2 & 200 & 252,67 & 50533,00 \\
& Total & 400 & & \\
\hline
\end{tabular}




\begin{tabular}{lr}
\hline \multicolumn{2}{c}{ Estadísticos de prueba $^{\text {a }}$} \\
\hline & \multicolumn{1}{c}{ Preocupación } \\
U de Mann-Whitney & 9567,000 \\
W de Wilcoxon & 29667,000 \\
Z & $-9,029$ \\
Sig. asintótica(bilateral) &, 000 \\
a. Variable de agrupación: Grupo & \\
\hline
\end{tabular}

El valor del estadístico $U$ de Mann Whitney con $\mathrm{p}$ valor $<0,05$, nos indica que hay diferencias importantes en el grado de preocupación ante los examenes de los alumnos de las dos Instituciones educativa privadas en estudio, de la institución educativa privada 1 presentó un (Promedio $=135.25)$ a diferencia de la ansiedad ante los exámenes de la institución educativa privada 2 , con un (Promedio $=270.76$, afirmando que existe diferencias significativas de los niveles de preocupación en estudiantes de secundaria de dos Instituciones Privadas.

Tabla 4.

Contrastación de hipótesis de comparación del nivel de reacciones fisiológicas en estudiantes de secundaria de dos Instituciones Privadas

\begin{tabular}{llrrr}
\hline \multicolumn{4}{c}{ Rangos } \\
\hline & Grupo & N & Rango promedio & Suma de rangos \\
\hline Reacciones fisiológicas & I.E privada 1 & 200 & 132,08 & 26416,00 \\
& I.E privada 2 & 200 & 268,92 & 53784,00 \\
& Total & 400 & & \\
\hline
\end{tabular}

\begin{tabular}{lr}
\hline \multicolumn{2}{c}{ Estadísticos de prueba $^{\text {a }}$} \\
& $\begin{array}{l}\text { Reacciones } \\
\text { fisiológicas }\end{array}$ \\
\hline U de Mann-Whitney & 6316,000 \\
W de Wilcoxon & 26416,000 \\
Z & $-11,842$ \\
Sig. asintótica(bilateral) &, 000 \\
a. Variable de agrupación: Grupo & \\
\hline
\end{tabular}

El valor del estadístico $U$ de Mann Whitney con $\mathrm{p}$ valor $<0,05$, nos indica que hay diferencias importantes en las reacciones fisiológicas de los estudiantes de las dos Instituciones educativa privadas en estudio, son los estudiantes de la institución educativa privada 1 presentó un (Promedio $=132.08$ ) a diferencia de las reacciones fisiológicas de la institución educativa privada 2 , con un (Promedio $=268.92$ ), afirmando que existe diferencias significativas de los niveles de reacciones fisiológicas en estudiantes de secundaria de dos Instituciones Privadas,

Tabla 5.

Contrastación de hipótesis de comparación del nivel de situaciones ante los exámenes estudiantes de secundaria de dos Instituciones Privadas

\begin{tabular}{llrrr}
\hline \multicolumn{5}{c}{ Rangos } \\
\hline & Grupo & N & Rango promedio & Suma de rangos \\
\hline Situaciones & I.E privada 1 & 200 & 139,89 & 27978,00 \\
& I.E privada 2 & 200 & 261,11 & 52222,00 \\
& Total & 400 & & \\
\hline
\end{tabular}

\section{Estadísticos de prueba ${ }^{\mathrm{a}}$}




\begin{tabular}{lr}
\hline & Situaciones \\
\hline U de Mann-Whitney & 7878,000 \\
W de Wilcoxon & 27978,000 \\
Z & $-10,490$ \\
Sig. asintótica(bilateral) &, 000 \\
a. Variable de agrupación: Grupo & \\
\hline
\end{tabular}

El valor del estadístico $U$ de Mann Whitney con $\mathrm{p}$ valor $<0,05$, nos indica que hay diferencias importantes en situaciones ante los exámenes de los alumnos de las dos Instituciones educativa privadas en estudio, son los estudiantes de la institución educativa privada 1 presentó un (Promedio $=139.89$ ) a diferencia de las situaciones de la institución educativa privada 2, con un (Promedio $=261.11$ ), afirmando que existe diferencias significativas de los niveles de situaciones en estudiantes de secundaria de dos Instituciones Privadas.

Tabla 6.

Contrastación de hipótesis de comparación del nivel de reacciones de evitación estudiantes de secundaria de dos Instituciones Privadas,

\begin{tabular}{llrrr}
\hline \multicolumn{4}{c}{ Rangos } \\
\hline & Grupo & N & Rango promedio & Suma de rangos \\
\hline Reacciones de evitación & I.E privada 1 & 200 & 133,22 & 26644,50 \\
& I.E privada 2 & 200 & 267,78 & 53555,50 \\
& Total & 400 & & \\
\hline
\end{tabular}

\begin{tabular}{lr}
\hline \multicolumn{2}{c}{ Estadísticos de prueba $^{\text {a }}$} \\
\hline & $\begin{array}{c}\text { Reacciones de } \\
\text { evitación }\end{array}$ \\
\hline U de Mann-Whitney & 6544,500 \\
W de Wilcoxon & 26644,500 \\
Z & $-11,655$ \\
Sig. asintótica(bilateral) &, 000 \\
a. Variable de agrupación: Grupo & \\
\hline
\end{tabular}

El valor del estadístico $U$ de Mann Whitney con $\mathrm{p}$ valor $<0,05$, nos indica que hay diferencias importantes en el nivel de reacciones de evitación de los alumnos de las dos Instituciones educativa privadas en estudio, son los alumnos de la Institución Educativa Privada 1 presentó un (Promedio $=133.22$ ) a diferencia de las reacciones de evitación de la institución educativa privada 2 , con un (Promedio $=267.781$ ), afirmando que existe diferencias significativas de los niveles de reacciones de evitación en estudiantes de secundaria de dos Instituciones Privadas.

\section{Discusión}

En relación a la hipótesis planteada: Se encuentran diferencias importantes entre los niveles de ansiedad ante algún examen en alumnos de secundaria de dos Instituciones Educativas Privadas, se identificó que de acuerdo con los encuestados y a los resultados encontrados, Institución Educativa Particular se observó en la institución educativa privada 1, que, el $29 \%$ presenta nivel leve, el $70 \%$ presenta nivel moderado y el $1 \%$ presenta nivel grave y en cuanto a la institución educativa privada 2 , el $1.5 \%$ presentó nivel leve, el $71.5 \%$ presentó nivel moderado y el $27 \%$ presentó nivel grave de la variable ansiedad ante los exámenes. Al respecto, Torrano, Ortigosa, Riquelme, y López (2017) afirman que el miedo está relacionado con los centros educativos y se dan principalmente cuando culmina la niñez y tiene una continuidad cuando las personas son adolescentes. Al hablar de estos estados emocionales en las evaluaciones dentro del entorno educativo, 
se da generalmente la ansiedad en algún examen, y que define una característica específica y situacional de las personas que comprometen su rendimiento académico. se caracteriza en la predisponer a que reacciones con alta ansiedad en entornos que se relacionan al rendimiento. Para Torrecillas y Segura (2017) $\mathrm{La}$ ansiedad ante las evaluaciones, impactan en su rendimiento académico, creando además frustración miedo pudiendpo alterar su estabilidad emocional.

Se concluye que existen diferencias entre los valores obtenidos en los niveles de ansiedad por género en las Instituciones de estudio. Siendo las mujeres las que presentan mayores niveles de ansiedad. Asimismo, Sarason determina como componentes cognitivos de la ansiedad, las expectativas, las creencias y experiencias personales, las construcciones y las autovaloraciones sobre la idoneidad de confrontación (Domblás, 2016). Ésta se encuentra dentro del enfoque cognitivo conductual de la ansiedad, el cual, profundiza en el papel que desempeñan las variables cognitivas, teniendo en cuenta dos perspectivas diferentes. Por un lado, los procesos cognitivos ocasionan una respuesta de ansiedad y, por otro lado, esta situación de ansiedad incide en los procesos cognitivos y modifica el comportamiento. Ello constituye un claro problema en el sistema educativo peruano, dado que los estudiantes valoran como amenazante los eventos evaluativos que forman parte de su cotidianidad, por ejemplo: formular preguntas en clase, emitir opiniones ante un tema específico a modo evaluación oral o rendir exámenes escritos; y de alguna manera evitan recurrentemente la exposición a éstos (Furlán, 2013). La ansiedad ante los exámenes no solo constituye un grave problema por lo mencionado anteriormente, sino porque influye negativamente sobre su rendimiento académico (Valdez, Becerra, Melgarejo y Huaranca, 2019).

En relación a los niveles de ansiedad ante los exámenes, en su dimensión preocupación, según los resultados obtenidos del procesamiento del instrumento aplicado, se observó en la institución educativa privada 1, que, el 33\% presenta nivel leve, el $63 \%$ presenta nivel moderado y el $4 \%$ presenta nivel grave y en cuanto a la institución educativa privada 2, el $8 \%$ presentó nivel leve, el $63 \%$ presentó nivel moderado y el $29 \%$ presentó nivel grave de la dimensión preocupación ante los exámenes. Respecto de la base teórica que fundamenta la presente investigación, inicialmente de la variable ansiedad ante los examenes: La Teoría de la interferencia cognitiva (CIT) de Sarason, fue desarrollado para explicar la asociación entre la ansiedad y el rendimiento cognitivo reducido, manifestando que la ansiedad interfiere en los pensamientos de la persona por lo que sus preocupaciones impactan negativamente sobre su nivel de atención y ello conlleva a un rendimiento cognitivo adverso (Yockey y Henry, 2019).

Por otro lado, en los niveles de ansiedad ante los exámenes, en su dimensión reacciones fisiológicas, según los resultados obtenidos del procesamiento de datos obtenido, se observó que en la institución educativa privada 1, que, el 34.5\% presenta nivel leve, el $65 \%$ presenta nivel moderado y el $0.5 \%$ presenta nivel grave y en cuanto a la institución educativa privada 2 , el $7 \%$ presentó nivel leve, el $69 \%$ presentó nivel moderado y el $24 \%$ presentó nivel grave de la dimensión reacciones fisiológicas. Para Valle, Betegón,y Irurtia (2018) La ansiedad es una emoción y, por lo tanto, se presenta con experiencias subjetivas, cuando se activa psicofisiológica y en el comportamiento. En sí misma, es una respuesta adaptativa; pero si se presenta en el momento equivocado, o con un nivel de intensidad inadecuado, puede resultar disruptiva, perjudicar los procesos cognitivos, interferir con la adaptación al ambiente y afectar el rendimiento académico. Así, un estudiante ansioso debido a su aprendizaje de una lengua extranjera, es temeroso 
de ser participativo en clase, especialmente si de hablar en dicho idioma se trata, tienden a equivocarse y sentirse incomprendidos por sus expresiones en inglés, ello indica, una autoevaluación negativa como si tuviesen menos competencias en comparación con sus compañeros (Jarie et al., 2017); . Sobre la ansiedad ante los exámenes, ésta se aprecia en individuos intransigentes con sus equivocaciones, quienes incurren en el constante temor a por fallar en las respuestas a resolver en un examen, cuyo aspecto relevante, se debe tener en cuenta una serie de actividades que se manifiestan en la estructura de los exámenes de lengua extranjera, asimismo, esto presenta que los estudiantes más preparados puedan cometer equivocaciones (Nilsson, 2019).

Asismismo, existen diferencias significativas en los niveles de ansiedad ante los exámenes, en su dimensión situaciones, según los resultados obtenidos, se observó que en la institución educativa privada 1, que, el $30 \%$ presenta nivel leve, el $68 \%$ presenta nivel moderado y el $2 \%$ presenta nivel grave y en cuanto a la institución educativa privada 2, el 3.5\% presentó nivel leve, el $69.5 \%$ presentó nivel moderado y el $27 \%$ presentó nivel grave de la dimensión situaciones ante los exámenes. Es así que Rodríguez, Canedo, y Fernández (2019) definen este término como una situación en donde la persona ante los exámenes se siente amenazada, en el cual logra experimentar preocupación o que va a fracasar, a eso se le suma temas fisiológicos, emocionales los cuales se elevan de manera exponencial a lo habitual. De acuerdo a lo mencionado la ansiedad se experimenta como un elemento emocional en la cual se puede observar dos dimensiones la emocional" y "preocupación", que desarrollan tres manifestaciones, "cognitiva", "emocional" y "fisiológica", que le dan un carácter de constructo genérico.

Finalmente, existen diferencias significativas en los niveles de ansiedad ante los exámenes, en su dimensión reacciones de evitación, en estudiantes de secundaria, los resultados obtenidos del procesamiento del instrumento aplicado, fueron : la institución educativa privada 1, que, el $37.5 \%$ presenta nivel leve, el $60 \%$ presenta nivel moderado y el $2.5 \%$ presenta nivel grave y en cuanto a la institución educativa privada 2, el $1.5 \%$ presentó nivel leve, el $76 \%$ presentó nivel moderado y el $22.5 \%$ presentó nivel grave de la dimensión reacciones de evitación.Según Acevedo, Arenas, y Calderon (2020). Como resultado de un gran número de investigaciones realizadas a nivel mundial que comprueban la existencia de dificultades emocionales frente a las matemáticas en personas de diversas edades y niveles de formación, se acuñó el término ansiedad matemática. La ansiedad es una situación especial que se presenta cuando se conjugan factores de personalidad, ambientales e intelectuales. Entre los factores personalidad se encuentran la baja autoestima y el temor para preguntar; entre los ambientales, las experiencias negativas en el aprendizaje de las matemáticas, y las actitudes negativas tanto de padres de familia como de profesores; y entre los intelectuales, la sensación de incompetencia para aprender matemáticas, la falta de utilidad percibida de las matemáticas y la no coordinación entre los estilos de enseñanza de los profesores y los estilos de aprendizaje de los estudiantes.

\section{Referencias}

Acevedo, G. V., Arenas, T. Y., \& Calderon, W. J. (2020). Relación entre ansiedad matemática y rendimiento académico en matemáticas en estudiantes de secundaria / Relationship between mathematical anxiety and academic performance in 
mathematics in high school students. Ciencias Psicológicas, 4(1). doi:https://doi.org/10.22235/cp.v14i1.2174

Bausela ,E. (2005). Ansiedad ante los exámenes: evaluación e intervención psicopedagógica. Educere, 9(31),553-558. ISSN: 1316-4910. Recuperado de https://www.redalyc.org/articulo.oa?id=356/35603117

Boehme, K. L., Goetz, T., \& Preckel, F. (2017). Is it good to value math? Investigating mothers' impact on their children's test anxiety based on control-value theory. Contemporary Educational Psychology, 51, 11-21. doi: 10.1016/j.cedpsych.2017.05.002

Cassady, J. C., \& Johnson, R. E. (2002). Cognitive test anxiety and academic performance. Contemporary Educational Psychology, 27(2), 270-295. doi: 10.1006/ceps.2001.1094

Casari, L. M., Anglada, J., \& Daher, C. (2014). Estrategias de afrontamiento y ansiedad ante exámenes en estudiantes universitarios. Revista de Psicología, 32(2), 243269.

Delgado-Monge, I., Castro-Martínez, E., \& Pérez-Tyteca, P. (2020). Estudio comparativo sobre ansiedad matemática entre estudiantes de Costa Rica y España / Comparative study on mathematical anxiety among students from Costa Rica and Spain. Revista Electrónica Educare, 24(2), 1-21. doi:http://doi.org/10.15359/ree.24-2.15.

Domblás, A. (2016). Ansiedad, personalidad y rendimiento académico en alumnado de educación primaria. Pamplona: Universidad Pública de Navarra. Recuperado de https://academicae.unavarra.es/bitstream/handle/2454/20028/Tesis\%2bAndr\%c3 $\% a 9 s \% 2 b D o m b 1 \%$ c3\%a1s_ma.pdf?sequence $=1 \&$ isAllowed $=y$

Dominguez-Lara, S. (2016). Inventario de la ansiedad ante exámenes-estado: análisis preliminar de validez y confiabilidad en estudiantes de psicología. Liberabit, 219228.

Francisquelo, M. N., \& Furlan, L. (2016). Ansiedad ante los exámenes y rasgos de personalidad en estudiantes universitrios. Revista Argentina de Ciencias del comportamiento.

Furlan, L. A., Rosas, J. S., Heredia, D., Piemontesi, S., \& Illbele, A. (2009). Estrategias de aprendizaje y ansiedad ante los exámenes en estudiantes universitarios. Pensamiento Psicológico, 5(12), 117-124.

Furlán, L. (2013). Eficacia de una intervención para disminuir la ansiedad frente a los exámenes en estudiantes universitarios argentinos. Revista colombiana de $\begin{array}{lllll}\text { psicología, } & 22 & \text { (1), } & 75-89 . & \text { Recuperado de }\end{array}$ http://www.redalyc.org/articulo.oa?id=80428081006.

Jarie, L., Salavera, C., Teruel, P., y Salillas, J. (2017). La ansiedad lingüística en el aprendizaje del francés en alumnos de Enseñanza Secundaria Obligatoria. Cédille Revista de estudios franceses (13), 243-261. https://dialnet.unirioja.es/servlet/articulo?codigo=5974805 
Gutiérrez-Calvo, M. (1984). Ansiedad evaluativa y deterioro del rendimiento. Los mediadores cognitivos directos. Revista de Psicología General y Aplicada, 39(5), 963-982.

Gutierrez-Calvo, M. (1986). Influencia de las condiciones evaluativas sobre la accesibilidad de representaciones aversivas. Revista de Psicología General y Aplicada, 41(3), 565-583.

Hernández, R., y Mendoza, C. P. (2018). Metodología de la investigación. Las rutas cuantitativa, cualitativa y mixta. México D.F.: McGraw Hill Education.

Inglés, C. J., Piqueras, J. A., García-Fernandez, J. M., García-López, L. J., Delgado, B.,\& Ruiz-Esteban, C. (2010). Diferencias de género y edad en respuestas cognitivas, psicofisiólogicas y motoras de ansiedad social en la adolescencia. Psicothema, 22(3), 376-381.

Jiménez, M. A., y Ramírez, F. A. (2017). Inflación Subyacente en la República Dominicana : Medición y Evaluación. Alemania: MPRA Paper 84596, Biblioteca Universitaria de Munich.

Lohbeck, A., Nitkowski, D., \& Petermann, F. (2016). A control-value theory approach: relationships between academic self-concept, interest, and test anxiety in elementary school children. Child Youth Care Forum, 45(6), 887-904. doi: $10.1007 / \mathrm{s} 10566-016-9362-1$

Miguel-Tobal, J. J. (1996). La ansiedad. Madrid: Santillana.

Nilsson, M. (2019). Foreign language anxiety: The case of young learners of English in Swedish primary classrooms. Apples: Journal of Applied Language Studies, 13(2), 1-21. Recuperado de https://jyx.jyu.fi/bitstream/handle/123456789/63363/URN\%3aNBN\%3afi\%3ajy u-201902191584.pdf? sequence $=1 \&$ isAllowed $=\mathrm{y}$

Orgilés, M., Méndez, X., Espada, J. P., Carballo, J. L., \& Piqueras, J. A. (2012). Síntomas de trastornos de ansiedad en niños y adolescentes: diferencias en función de la edad y el sexo en una muestra comunitaria. Revista de Psiquiatría y Salud Mental, 5(2), 115-120. doi: 10.1016/j.rpsm.2012.01.005.

Putwain, D. W., \& Aveyard, B. (2018). Is perceived control a critical factor in understanding the negative relationship between cognitive test anxiety and examination performance? School Psychology Quarterly, 33(1), 65-74. doi: $10.1037 / \mathrm{spq} 0000183$.

Rodríguez, C. F., Canedo, M. d., \& Fernández, S. (2019). Ansiedad ante los exámenes en estudiantes de educación primaria: diferencias en función del curso y del género / Text anxiety in elementary school students: diferences by school year and gender. Publicaciones, 49(2), 151-168. doi::10.30827/publicaciones.v49i2.8087

Rodríguez, C. F., Canedo, M. d., Cerviño, S. F., \& Enríquez, E. B. (2019). NAsiedad ante los exámenes en estudiantes de educación primaria: diferencias en función del curso y del género. Publicaciones, 49(2), 151-168. doi:10.30827/publicaciones.v49i2.8087 
Sánchez, P. (2008). Psicología clínica. México D.F.: Manual Moderno.

Sandín, B. (2004). Miedos y fobias en la infancia y en la adolescencia. En Buendía J. (Ed.) Psicopatología en niños y adolescentes (pp. 147-161). Madrid: Pirámide.

Sandin, B., Chorot, P., Valiente, R. y Lostao, L. (2009). Validación española del cuestionario de preocupación PSWQ: Estructura factorial y propiedades psicométricas. Revista de Psicopatología y Psicología Clínica. (14). Recuperado de http://revistas.uned.es/index.php/RPPC/article/view/4070

Segool, N. K., von der Embse, N. P., Mata, A. D., \& Gallant, J. (2014). Cognitive behavioral model of test anxiety in a high-stakes context: An exploratory study. School Mental Health, 6(1), 50-61. doi: 10.1007/s12310-013-9111-7

Sierra, J. C., Ortega, V., \& Zubeidat, I. (2003). Ansiedad, angustia y estrés: tres conceptos a diferenciar. Revista Mal-Estar e Subjetividade, 3(1), 10-59.

Steinmayr, R., Crede, J., McElvany, N., \& Wirthwein, L. (2016). Subjective well-being, test anxiety, academic achievement: testing for reciprocal effects. Frontiers in Psychology, 6:1994. doi: 10.3389/fpsyg.2015.01994

Torrano, R., Ortigosa, J. M., Riquelme, A., \& López-Pina, J. A. (2017). Evaluación de la ansiedad ante los exámenes en estudiantes de educación secundaria obligatoria. Revista de Psicología clínica con niños y adolescentes, 4(2), 103-110. Obtenido de https://www.redalyc.org/pdf/4771/477152556002.pdf

Torrecillas, J. J., \& Segura, C. L. (2017). Ansiedad ante los exámenes moderada por la participación en actividades físicas y deportivas. INFAD Revista de Psicología, 4(1), 277-283. doi:https://doi.org/10.17060/ijodaep.2017.n1.v4.1057

Valdez A., Becerra, N., Melgarejo, B., y Huarancca, J. (2019). Nivel de ansiedad en adolescentes de una institución preuniversitaria de Lima Norte. Health Care \& Global Health, 3 (1), 12-6.

Valero, L. (1999). Evaluación de la ansiedad ante los exámenes: datos de aplicación y fiabilidad de un cuestionario CAEX. Anales de Psicología, 15(2), 223-231.

Valle, M. d., Betegón, E., \& Irurtia, M. J. (2018). Efecto del uso de estrategias cognitivas de regulación emocional sobre la ansiedad en adolescentes españoles. SUma Psicología, $25(2)$, $153-161$.

doi: http://dx.doi.org/10.14349/sumapsi.2018.v25.n2.7

Yockey, J., y Henry, M. (2019). Simulation Anxiety across the Curriculum. Simulación clínica en enfermería, 29, 29-37. Recuperado de https://scihub.do/https://www.sciencedirect.com/science/article/abs/pii/S18761399183023 17 